[DOI: 10.24214/jecet.A.10.4.61122]

Jaurnal of Environmental Science, Computer Science and Engineering \& Technology

An International Peer Review E-3 Journal of Sciences and Technology

Available online at www.jecet.org

Section A: Environmental Science

Research Article

\title{
Analyse De L'incidence Et La Sévérité De La Maladie Du Sommet Touffu (B.B.T.D.) Des Bananiers Cultives En Case Dans La Ville De Kindu Et Ses Hinterlands
}

\author{
SHADARI SALUMU AI hadid, MWEMEDI SIMAUNDU Cinto SOSTHÈNE \\ KITABY K., MUBWANA SHABANI César.
}

Département de Phytotechnie, Faculté des Sciences Agronomiques, Université de Kindu (UNIKI/Maniema/RD Congo).Université de Kindu UNIKI, République Démocratique du Congo

(RDC)/ ville de Kindu province du Maniema

Received: 21 Août 2021; Revised: 20 Septembre 2021; Accepted: 23 Octobre 2021

\begin{abstract}
Résumé : Cette étude avait comme objectif général d'Analyser l'incidence et la sévérité de la maladie du sommet touffu (B.B.T.D.) Cas de bananiers cultivés en case dans la ville de Kindu et ses hinterlands. En effet, elle avait comme objectifs spécifiques de :Déterminer le niveau d'attaque suivant l'échelle de cotation de BBTD sur les différents cultivars de bananiers cultivés à Kindu et ses hinterlands ;Identifier les cultivars résistants et sensibles par rapport à l'incidence et la sévérité de BBTD ;Préciser les sites dans le milieu d'étude le plus attaqué par le BBTD .En vue d'atteindre ces objectifs, nous avons utilisé la méthode analytique et comparative. En effet, la récolté des données a été rendu possible à l'aide de l'échelle de cotation de BBDV que nous avons utilisé.Les résultats obtenus ont montré que : L'incidence de BBTV dans la zone d'étude a été plus remarquée dans les hinterlands de la ville de Kindu. Cependant, l'incidence la plus élevée était notée aux sites : PK16 Kalemba route Lokando avec 58,71\%, suivi de PK10 Misenge avec 54,85\% et PK7 Katako avec 53, 16\%.Par contre, cinq (5) cultivars ont été identifiés Kebobo, Mbudi, Muyahudi, Ndogila ET Nsekumuna. Ainsi, le cultivar Kebobo présente un taux plus élevé respectivement avec 48,6\% et le cultivar Mfuba Ndogila avec 47,87\%.La sévérité de BBTV dans la zone d'étude était abondant au niveau 2 (stries foncées jusqu'au pseudotronc) et niveau 4 (taille réduite de feuilles décolorées) ; néanmoins, les sites Alunguli Centre, Alunguli Sud et PK7 Katako route Kibombo ont présenté respectivement un
\end{abstract}


taux de sévérité plus élevée respectivement avec 95,5\% au niveau 3 et 86,66\% niveau 4 et $64,58 \%$ niveau 4.Ainsi, le cultivar ayant présenté un taux d'incidence la plus élevée par rapport à d'autres était le cultivar Nsekumuna avec 44\% et une sévérité de 39,57\% suivi des autres.A ce niveau les cultivateurs peuvent utiliser les cultivars Mbudi et Muyahudi Car ils ont été légèrement résistants au BBTD par rapport aux autres cultivars.

Mots clés : incidence, sévérité, maladie, cultivars, bananier,

\section{INTRODUCTION}

Le Bananier cultivé (bananes et plantains) constitue une source alimentaire pour des millions des personnes dans le monde. Sa culture s'étend sur plus de 120 pays des Régions Tropicales et Subtropicales à travers les cinq continents, ${ }^{[1]}$. La culture constitue un aliment de base et la source de revenu pour environ plus de 400 millions des personnes dans les pays en développement de l'Amérique du Sud, de Sud-Est Asiatique et de l'Afrique, ${ }^{[2]}$.

En Afrique la production annuelle des bananes plantains s'élève à 25 millions de tonnes soit $34 \%$ de la production mondiale où les régions importantes aux meilleurs rendements sont notamment les régions tropicales humides (Rd Congo, Congo Brazza, Gabon, Cameroun, Nigéria, Ghana, Côte d'ivoire, Guinée et au Libéria.). Plus de 90\% des Bananes produites en Afrique sont consommées localement, [3].

Plus de 70 millions de personnes dans 15 pays d'Afrique Subsaharienne dépendent de la banane pour leur approvisionnement alimentaire et leur bien-être. Leur sécurité alimentaire est menacée par la présence et la propagation de deux maladies dévastatrices pour la banane : la maladie de Bunchy top et Banana Xanthomonas Wilt. Une réunion internationale a été convoquée en août 2009 afin de trouver une solution à la propagation de cette crise régionale ${ }^{[4]}$

La maladie du sommet touffu est présente dans 11 pays en Afrique subsaharienne notamment Angola, Burundi, Cameroun, République Centrafricaine, Congo, République Démocratique du Congo, Guinée Equatoriale, Gabon, Malawi, Rwanda et Zambie. ${ }^{[5]}$

En République démocratique du Congo (R.D.C) ; la Banane plantain constitue une nourriture de base pour la population du Nord-Est et du Centre du Pays. Près de $70 \%$ de la production bananière s'y consomment directement par les producteurs locaux, ruraux, $30 \%$ restant représente la partie commerciale et l'ensemble après sa récolte, ${ }^{[6]}$.

Dans la Province du Maniema plus précisément à Kindu et ses environs, les bananes et les plantains constituent la troisième denrée alimentaire après le manioc et le maïs et jouent de ce fait un rôle très important dans la sécurité alimentaire et sur le revenu des producteurs,

Les bananiers sont cultivés dans les champs paysans souvent en association avec d'autres cultures (riz, manioc, mais, taro, igname ...) les grandes productions proviennent en amont et en aval du fleuve Congo, la route vers Kasongo, Kibombo, Kalima même en forêt secondaire vieille ou en jachères dans les environs de la ville. Dans le centre-ville, le bananier est en culture de case où ils sont plantés tout autour des maisons pour l'autoconsommation. ${ }^{[7]}$

Parmi le mode de consommation des bananes et des plantains, la cuisson sous diverses formes selon le régime alimentaire est une évidence dans la région. Après la cuisson, le plantain est consommé avec plusieurs légumes comme le feuilles de manioc, l'amarante, morelle amère, l'aubergine africaine etc. on consomme également avec la viande et le poisson. Comme aliment de lux, les plantains cuits sont 
pilés et consommé comme «LITUMA » la forme grillée est actuellement courante (vente en sachet de banane grillée). Ainsi la banane est utilisée comme un dessert. ${ }^{[8]}$

Malgré les avantages que relèvent la culture du Bananier dans cette région, sa production demeure toujours faible, chose qui affecte considérablement la loi de l'offre et de la demande sur le marché. Son absence ou sa carence lui fait un aliment précieux pour un certain nombre de la population surtout celle qui est soumise en régime alimentaire, alors que le bananier pouvait qu'être un aliment de base comme notamment le Manioc et maïs. ${ }^{[9]}$.

L'absence de filière d'approvisionnement en matériel sain et à haut rendement oblige les agriculteurs à se procurer des rejets tout venants pour leurs plantations. Mais l'utilisation de ce matériel végétal mal identifié et souvent parasité dans la conduite de la bananeraie engendre des difficultés importantes qui affectent les rendements ${ }^{[10]}$.

Parmi les nombreuses attaques des bananiers, celles des virus sont les plus redoutable car il n'existe aucun traitement curatif. Or, les maladies virales constituent un problème très répandu dans le monde végétal, causant d'importants dégâts. ${ }^{[1]}$

Les maladies à virus des bananiers ont une grande incidence physiologique, car elles entraînent une diminution de la vigueur des plantes et modifient bien souvent l'aspect de certains organes végétaux. [12]

Elles se différencient assez bien des autres maladies mais l'identification de l'agent pathogène en cause ne peut être vérifiée que par la mise en œuvre de méthodes de diagnostic au laboratoire. Par contre, dans certaines situations le bananier est aussi attaqué par les champignons, les nématodes, les insectes ainsi que les causes abiotiques, ${ }^{[13]}$

L'une des infections virales la plus dévastatrice est le Banana Bunchy Top Disease (BBTD) qui continue son expansion dans le monde. Cette maladie est causée par le virus de Bunchy Top du bananier (BBTV) qui est la maladie la plus importante et la plus dévastatrice dans de nombreux pays tropicaux. Dans de nombreuses zones, la production et la superficie des bananiers sont réduites de $90-95 \%$, ${ }^{[14]}$. Les virus sont transmis par l'aphide Pentalonia nigronervosa. La maladie est reconnue par les symptômes suivants : les nanismes de plantes, le rabougrissement de feuilles, présence de stries, réduction de la taille de feuille et leurs décolorations au bordure, forme du sommet touffu (on dirait le balai de sorcier). [15]

A l'issu de cette étude, nous nous sommes fixé comme objectifs spécifiques :

- Déterminer le niveau d'attaque suivant l'échelle de cotation de BBTD sur les différents cultivars de bananiers cultivés à Kindu et ses hinterlands ;

- Identifier les cultivars résistants et sensibles par rapport à l'incidence et la sévérité de BBTD ;

- Préciser les sites dans le milieu d'étude le plus attaqué par le BBTD ;

Pour atteindre ces objectifs, l'étude s'est proposé de vérifier les hypothèses suivantes :

> Le niveau d'attaque de BBTD serait très élevé sur les différents cultivars de bananiers cultivés à Kindu et ses environs ;

$>$ Le cultivar Muyaudi et Mbudi pourraient être identifié comme étant résistant au BBTD dans le milieu ;

> Le taux de rendements perdus pourrait être variable suivant les cultivars et périodes de plantation ; 
> Certains sites et villages de production de banane et de plantain seraient attaqués par la maladie de sommet touffu de bananier chose qui pouvait justifier la non fréquence de produit sur les marchés locaux.

\section{MATERIEL ET METHODES}

Notre étude a été réalisée dans la ville de Kindu et ses hinterlands et cela pendant la période du 12/2/2020 jusqu' au 12/05/2020, soit 4 mois d'études; La Ville de Kindu est le Chef-lieu de la Province du Maniema, une jeune ville-urbano-rural de 33 ans si on la compare à d'autres anciennes villes. Elle est constituée de trois communes ; entre autres Commune de Mikelenge et de Kasuku qui sont situées à la rive gauche du fleuve Congo, ainsi que la Commune d'Alunguli qui se trouve à la rive droite du fleuve ; la ville de Kindu compte 9 quartiers (trois par commune). ${ }^{[16]}$

2.1. Localisation: La ville de Kindu est située sur les deux rives du fleuve Congo (ou Lualaba) avec une superficie de 10130ha. Elle est limitée au Nord par le territoire de Kailo, à l'Est par le territoire de Pangi, à l'Ouest par le territoire de KIBOMBO et au Sud par le territoire de Kasongo ; Elle est comprise entre $2^{\circ}, 57^{\prime}$ de latitude Sud et entre $24^{\circ} 55^{\prime}$ et $28^{\circ} 58^{\prime}$ de longitude Est, et une altitude de $472 \mathrm{~m},{ }^{[17]}$.

2.2. Climat: La Ville de Kindu a un climat tropical chaud et humide caractérisé par deux saisons : saison de pluie et saison sèche, La saison sèche commence de mi-mai jusqu'à la mi-août (saison $\mathrm{B}$ ) et la saison de pluie commence de mi-août jusqu'à la mi-mai (saison A), pendant la saison de pluie, il se remarque une petite saison sèche qui prend mi-janvier jusqu'à la mi-février. D'après Koppen et Geiger, le climat y est classé du type Aw3. Kindu affiche une température annuelle moyenne de $25.4^{\circ} \mathrm{C}$. Les précipitations annuelles moyennes sont de $1650 \mathrm{~mm}$, d'où les précipitations varient de $192 \mathrm{~mm}$ entre le plus sec et le plus humide des mois, ${ }^{[18]}$ (M'PIANA, 2010) Les données climatiques qui ont prévalues pendant la période de notre étude sont reprises dans le tableau ${ }^{\circ} 1$ ci-dessous. ${ }^{[16]}$

Tableau 1 : Paramètres météorologiques de la ville de Kindu

\begin{tabular}{llllll}
\hline $\begin{array}{l}\text { Paramètres } \\
\text { observer }\end{array}$ & à & \multicolumn{2}{c}{ Précipitation } & Pluviométrie & $\begin{array}{l}\text { Nombre de } \\
\text { pluie tombée } \\
\end{array}$ \\
& Minimale & optimale & Maximale & Eau tombée en mm & Jours de pluie \\
\hline Février & 22,6 & 27 & 36 & 131,8 & 6 \\
Mars & 22,4 & 26,1 & 36,2 & 168,2 & 12 \\
Avril & 21,5 & 25 & 36,1 & 57,4 & 5 \\
Mai & 20,9 & 26,02 & 36,4 & 101,4 & 7 \\
$\Sigma$ & 87,4 & 104,12 & 144,7 & 458,8 & 30 \\
$\dot{\text { X }}$ & 22 & 26 & 36 & 114 & 8 \\
\hline Source $:[19]$ & & & & &
\end{tabular}

2.3.Hydrographie : La ville est traversée par le majestueux fleuve Congo avec quelques petits affluents à la rive gauche comme à la rive droite : les rivières Mikonde, Misubu, Muchado, Mangobo, Kamabala, et Kamikunga à la rive droite ; ainsi que Mikelenge, Mukoloshi, Makopo, Lwandoko, Canon, Rukukuye et Ngwangwata à la rive gauche.

2.4.Végétation, sol et relief :Au Maniema, la forêt équatoriale décrit un arc de cercle partant de Kindu en passant par Shabunda et par Walikale pour rejoindre la forêt équatoriale du Sud de Béni. Partant du Sud de Kindu au Niveau du Sud d'uvira jusqu'à la limite avec la Province du Tanganyika. On trouve des mosaïques de savanes boisées et les galeries forestières traduisant la dégradation de la forêt par l'action de l'homme. La faune est riche et variée. La ville de Kindu a une végétation très variée, mais l'on y trouve surtout des arbres fruitiers et d'autres arbres plantés. Les blocs ruraux sont en partie 
couverts par une brousse où se pratique l'agriculture. Son sol varie entre les types argilo-sablonneux et sablo-argileux, Le relief est caractérisé par l'extrémité de la cuvette centrale congolaise. ${ }^{[20]}$

2.5. Matériels : Au cours de notre étude, les matériels qui ont été utilisé sont : la fiche de l'échelle de cotation de BBTD sur les cultivars suivants : Mbudi, Kebobo, Mfumba Ndongila, Nsekumana, et Muyaudi, l'appareil photographique, le carnet de récolte des données

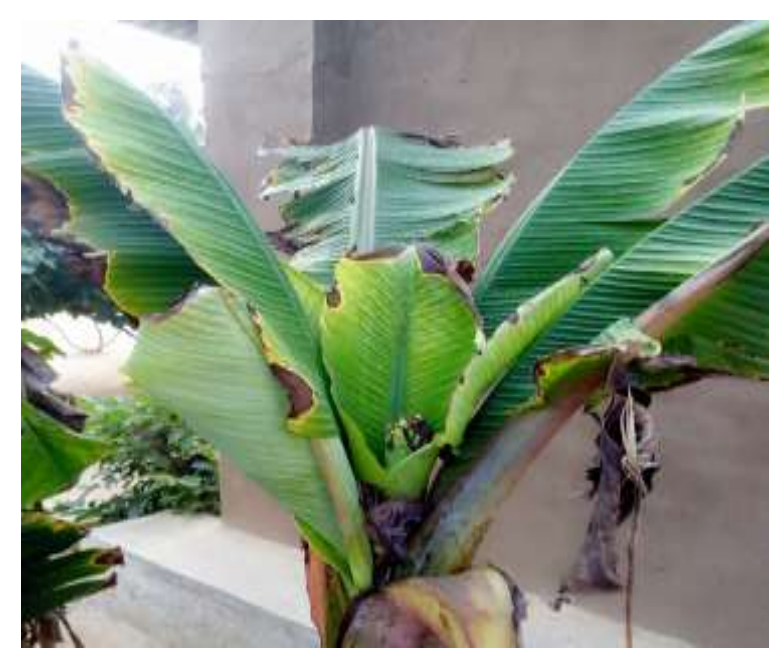

Photo $\mathbf{N}^{\circ} 1$ : aspect touffu au sommet « Bunchy top », var. MBUDI, (prise sur la place feue rouge)

2.6. Méthodes: Nous avons utilisé la méthode analytique et comparative. En effet, la récolté des données a été rendu possible à l'aide de l'échelle de cotation de BBDV que nous avons utilisé

\subsubsection{Conduite de la recherche}

Notre conduite a été réalisée dans les 3 classes en vue d'analyser l'incidence et la sévérité de BBTD à l'aide de l'échelle de cotation; et cela de la manière suivante :

- En date du 12/02/2020 au 13/02/2020, nous avions effectué une descente sur terrain pour le traitement T9 (Basoko, Rva) se trouvant dans la deuxième classe.

- En date du 14/02/2020, nous avions effectué une descente sur terrain pour le traitement T10 (PK7 Malonga) se trouvant dans la deuxième classe.

- En date du 18/02/2020 au 22/02/2020, nous avions effectué une descente sur terrain pour le traitement T12 (Brazza, Tokolote, Mission, Shenge) se trouvant dans la troisième classe.

- En date du 24/02/2020 au 28/02/2020, nous avions effectué une descente sur terrain pour le traitement T13 (Omata, Modernite) se trouvant dans la troisième classe.

- En date du 04/03/2020, nous avions effectué une descente sur terrain pour le traitement T6 (Katako, route Kibombo) se trouvant dans la deuxième classe.

- En date du 09/03/2020 au 15/03/2020, nous avions effectué une descente sur terrain pour le traitement T7 (Cite, Lumbulumbu, Tokedi, Du Plateau) se trouvant dans la deuxième classe.

- En date du 19/03/2020 au 08/04/2020, nous avions effectué une descente sur terrain pour le traitement T8 (Jamaica, 3z, Sncc, Handicape) se trouvant dans la deuxième classe. 
- En date du 12/04/2020, nous avions effectué une descente sur terrain pour le traitement T14 (Hewa Bora, Aeroport, Isc-Kindu-Feu Rouge) se trouvant dans la troisième classe.

- En date du 20/04/2020, nous avions effectué une descente sur terrain pour le traitement T15 (Pk10 Misenge) se trouvant dans la troisième classe.

- En date du 24/04/2020, nous avions effectué une descente sur terrain pour le traitement T11 (Pk16 Kalemba, route Lokando) se trouvant dans la troisième classe.

- En date du 05/05/2020, nous avions effectué une descente sur terrain pour trois traitements, dont T1 (Pk7 Mwene Kasile, route Kasongo) ; T2 (Alunguli Sud, Av. Kanabayonga) ; T3 (Alunguli Centre, Av. Penemisenga) se trouvant dans la première classe.

- En date du 12/05/2020, nous avions effectué une descente sur terrain pour deux traitements, dont T4 $\left(\mathrm{Km}^{2}=\right.$ Kilo Mbili, route Kalima) se trouvant dans la première classe.

L'observation a consisté à évaluer les plants des bananiers dans les champs et jardins de case. L'évaluation de la sévérité de la maladie sur la touffe infectée a été effectuée à l'aide de l'échelle de cotation de BBTVD (1-5) suivant :

$>$ Niveau 0 : sans symptôme

$>$ Niveau 1 : stries sur les feuilles,

$>$ Niveau 2 : stries foncées jusqu'au pseudo-tronc,

$>$ Niveau 3 : décoloration de feuilles avec taille normale,

$>$ Niveau 4 : taille réduite de feuilles décolorées,

$>$ Niveau 5 : aspect touffu au sommet « Bunchy top ${ }^{[15]}$.

\section{RESULTATS ET DISCUSSION}

Les résultats obtenus au cours de cette investigation sont présentés dans les figures ci-dessus suivi de leur interprétation

3.1. Evaluation d'incidence: symptômes de BBTD sur au moins un plant de la touffe: Cependant, la figure 15 ci-dessous montre la répartition de l'incidence de BBTD dans les différents sites ou traitements sur l'ensemble des cultivars de bananiers répertoriés

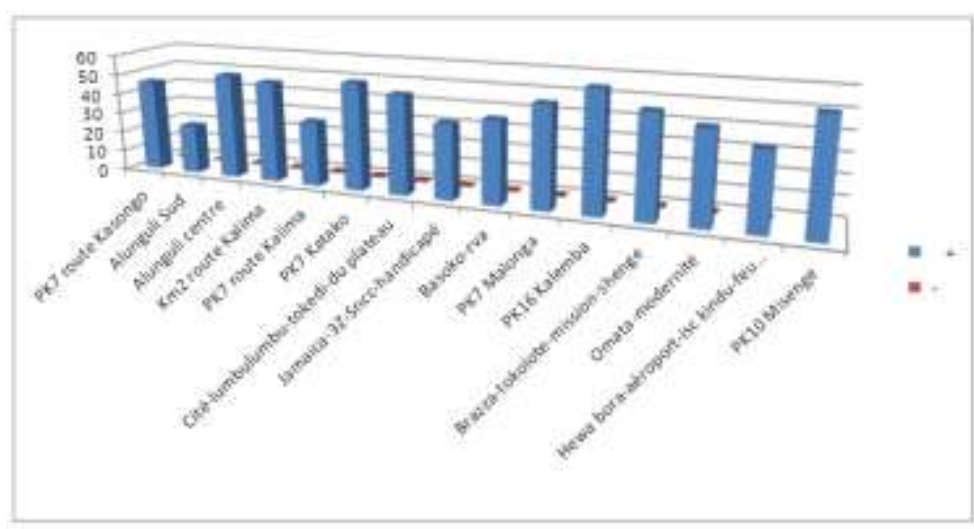

Figure $\mathbf{N}^{\circ} \mathbf{1}$ : Taux d'incidence de BBTV sur l'ensemble de la ville de Kindu et ses hinterlands

Au regard des résultats inscrits dans la figure $\mathrm{N}^{\circ} 1$ ci-haut, il s'observe que le taux d'incidence de BBTD le plus élevé était remarqué au site Pk16 village Kalemba, route Lokando avec 58,71\%, suivi du site 
Pk10 village Misenge avec 54,85\%, Pk7 village Katako, route Kibombo avec 53,16\%, et Alunguli Centre avec 52,23\% ; tandis que le taux d'incidence inférieur aux valeurs précitées ont été notées respectivement faibles et intermédiaires aux sites : Brazza-Tokolote-Mission-Shenge avec 50,24\%, Alunguli $\mathrm{Km}^{2}$ (Kilo Mbili) route Kalima avec 50,16\%, Pk7 Malonga avec 50\%, Cite-LumbulumbuTokedi-Du Plateau avec 49\%, Pk7 village Mwene Kasile, route Kasongo avec 46\%, Omata-Modernite avec 45\%, Basoko-Rva avec 40,81\%, Hewa Bora-Aeroport-Isc-Kindu-Feu Rouge avec 37,77\%, Jamaica-3z-Sncc-Handicape avec 36,96\%, PK7 village Lokongo, route Kalima avec 31,99\% et Alunguli Sud avec $24,44 \%$.

En effet, après interprétation des résultats inscrits dans la figure 15 ci-haut concernant l'incidence de BBTD, il s'explique que, le taux d'incidence le plus élevé était essentiellement trouvé dans les hinterlands de la ville de Kindu par rapport au centre-ville ; cette situation s'expliquerait du fait que, ces hinterlands se trouvent en milieux ruraux où se situent les forêts et brousses, le biotope des vecteurs de la maladie.

Aussi, faudra-t-il ajouter en disant que même dans l'expérimentation au champ, les plantes des bordures sont souvent plus attaquées tout en étant à côtés des mauvaises herbes qui constituent les réservoirs des insectes vecteurs et porteurs des maladies. Selon Caruana ${ }^{[14]}$, le commerce basé sur les échanges des rejets, a joué un rôle primordial dans la dissémination du virus de BBTD à travers le monde. Ceci dit, la faible incidence dans le centre-ville de Kindu est que, la ville est en train d'être approvisionnée en matériels infectés en provenance de ses hinterlands. Adrien Kalonji M. ${ }^{[21]}$, ajoute que, le transport de l'inoculum peut également se réaliser par les matériels de propagation (boutures, bulbes, tubercules, rhizomes, graines, etc.), les débris des végétaux, les pratiques culturales, les machines agricoles et autres outils lors des opérations culturales, les récoltes. Le même auteur parle que, l'homme assure la dispersion des germes pathogènes par les échanges fréquents des matériels, les voyages, la multiplication végétative, et par les importations et exportations des marchandises. $\mathrm{Ngama}^{[15]}$, ajoute que la dissémination sur une longue distance est faite par l'homme qui utilise des rejets provenant de plantations infectées. La dissémination sur une courte distance à partir de foyers d'infection est faite par les pucerons.

3.2. Taux d'incidence de BBTD suivant les différents cultivars répertoriés : En effet, les données relatives aux résultats de l'incidence de BBTD sur les différents cultivars de bananier répertoriés dans le milieu d'étude sont reprises dans la figure 2 ci-dessous.

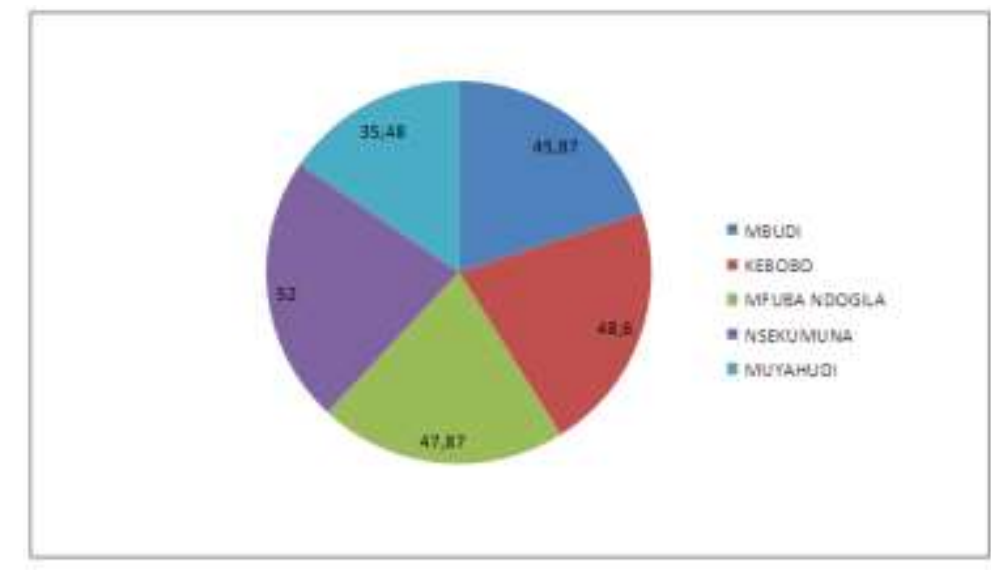

Figure $\mathbf{N}^{\circ} 2$ : Taux d'incidence de BBTD en \%, des différents cultivars de bananier répertoriés 
Il ressort de cette figure $\mathrm{N}^{\circ} 2$ que le taux d'incidence de BBTD le plus élevé, était observé au cultivar Kebobo soit 48,6\% d'incidence, il a été suivi des cultivars : Mfuba Ndogila avec 47,87\%, Mbudi avec $45,87 \%$, Nsekumuna avec 52\% et Muyahudi $35,48 \%$ d'incidence.

S'agissant des résultats de l'incidence de BBTD sur les différents cultivars répertoriés dans la ville de Kindu et ses hinterlands ; le taux d'incidence le plus élevé s'est fait observer au cultivar Kebobo avec $48,6 \%$; rien d'étonnant car la résistance aux maladies varie d'un cultivar à l'autre.

Selon Adrien Kalonji $\mathrm{M}^{[21]}$, rapporte que, en cas d'attaque importante du ravageur ou de l'agent pathogène, les variétés pourront développer quelques symptômes. Monde G., ${ }^{[2]} 2012$, dit de son côté que, les plantes montrent vis-à-vis des virus un mécanisme particulier de résistance qui est mis en évidence par le déclin de la teneur en virus lors d'une infection et le phénomène de rétablissement qui s'observent lors d'une infection. Adrien Kalonji M, ${ }^{[21]}$ 2008, ajoute en disant que, certains végétaux disposent des structures anatomiques physiques naturelles et d'autres ont développé des appareillages qui deviennent des barrières structurales nouvelles qui leur permettent de faire obstacle à l'action des parasites.

3.3. Cotation de la sévérité sur la touffe infectée suivant l'échelle de BBTD dans les différents sites de notre milieu d'étude : La figure 3 illustre la sévérité de la maladie sur les touffes infectées suivant l'échelle de cotation de BBTD (1-5).

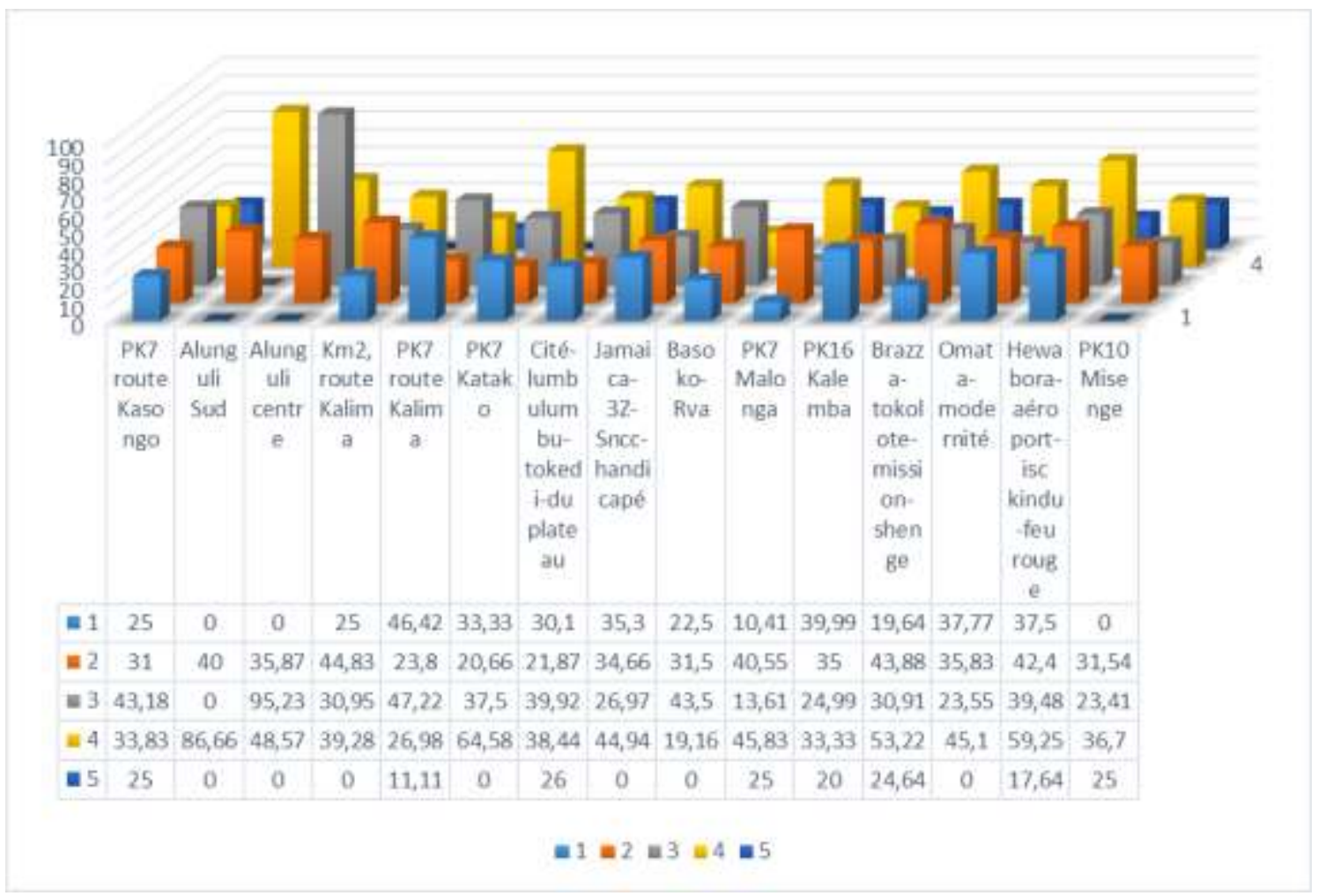

Figure $\mathbf{N}^{\circ} \mathbf{3}$ : La sévérité de BBTD dans la ville Kindu et ses hinterlands en \%.

De cette figure $\mathrm{N}^{\circ} 3$, il ressort en général que les niveaux de sévérité de BBTD les plus abondants dans le milieu d'étude sont occupés par le niveau 2 (stries foncées jusqu'au pseudo-tronc) et le niveau 4 (taille réduite de feuilles décolorées), suivi de niveau 3 (décoloration de feuilles avec taille normale), tandis que le niveau 1 et 5 ont été remarqué faiblement dans le milieu d'étude, stries sur les feuilles et aspect touffu au sommet « Bunchy top ». 
Cependant, le niveau 3 (décoloration de feuilles avec taille normale) le plus élevé a prédominé dans les sites Alunguli Centre avec un taux de 95,5\%, suivi des sites : Pk7 village Lokongo avec 47,22\%, Basoko-Rva avec 43,5\%, Pk7 village Mwene Kasile avec 43,18\% et Cite-Lumbulumbu-Tokedi-Du Plateau avec 39,92\%, tandis que le niveau 4 (taille réduite de feuilles décolorées) a prédominé aussi dans les sites: Alunguli Sud avec 86,66\%, Pk7 Katako route Kibombo avec 64,58\%, Hewa BoraAeroport-Isc-Kindu-Feu Rouge avec 59,25\%, Brazza-Tokolote-Mission-Shenge avec 53,22\%, Pk7 Malonga avec 45,83\%, Omata-Modernite avec 45,1\%, Jamaica-3z-Snnc-Handicape avec 44,94\% et Pk10 Misenge avec 36,7\%. Par ailleurs, le niveau 2 (stries foncées jusqu'au pseudo-tronc) était plus élevé seulement au site $\mathrm{Km}^{2}$ route Kalima avec un taux de $44,83 \%$ quelques soit son abondance dans tous les sites. Et enfin, le niveau 1(stries sur les feuilles) le plus élevé était obtenu au site Pk16 Kalemba, route Lokando avec 39,99\%.

Selon Roland ${ }^{[23]}$, rapporte que, les symptômes dû aux virus chez les végétaux peuvent varier et prendre des aspects différents selon les conditions du milieu ; l'apparition des symptômes constituent le résultat de l'intensité de la réaction de la plante à l'attaque d'un virus qui pourra être différente suivant le milieu rapporte.

Après analyse des résultats inscrits dans la figure 4, il s'est observé que le taux de sévérité de BBTD suivant l'échelle de cotation a été réparti de manière disparate selon les différents sites (ville de Kindu et ses hinterlands).

3.5. Taux de sévérité moyen des différents cultivars identifiés dans le milieu d'étude : En effet, les données relatives aux résultats de la sévérité de BBTD sur les différents de bananier répertoriés dans le milieu d'étude sont reprises dans la figure 4 ci-dessous.

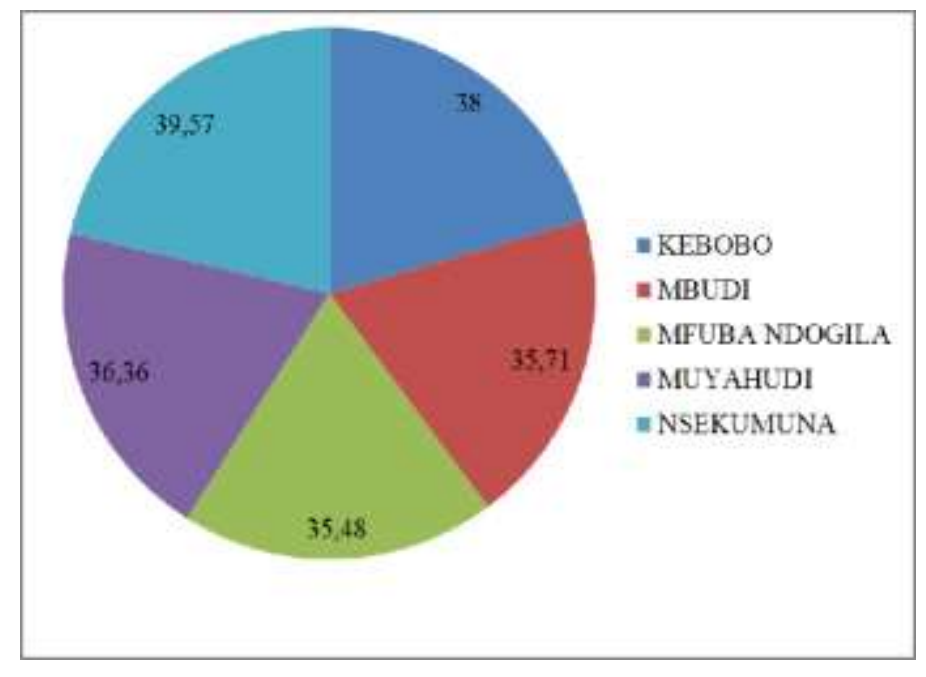

Figure $\mathbf{N}^{\circ} 4$ : Taux de sévérité de BBTD des différents cultivars de bananier répertoriés, en \%

Il ressort de cette figure $\mathrm{N}^{\circ} 4$ que, le taux de sévérité de BBTD le plus élevé était observé au cultivar Nsekumuna soit 39,57\% de sévérité ; il a été suivi des cultivars : Kebobo avec 38\%, Mbudi avec 35,71\%, Mfuba Ndogila avec 35,48\% et Muyahudi avec 36,36\% de sévérité.

Selon Thomas et al., rapporte que, les plants peuvent être infectés à tous les stades de croissance et il existe quelques différences initiales entre les symptômes développés par un plant infecté par puceron et ceux d'un plant issu de matériel infecté. 
Le même auteur continu à dire que, sur le plant infecté très tardivement dans le cycle de culture, le seul symptôme présent peut être quelques stries vert-foncées à l'extrémité de la bractée florale.

\section{CONCLUSION ET SUGGESTION}

A l'issu de cette étude, qui a portait sur Analyse de l'incidence et la sévérité de la maladie du sommet touffu (B.B.T.D.) Cas de bananiers cultivés en case dans la ville de Kindu et ses hinterlands

Afin de vérifier ces hypothèses, nous avons fait recours à la méthode analytique en s'appuyant sur la technique d'échantillonnage, dans un dispositif hiérarchisé placé dans l'ensemble de la ville de Kindu et ses hinterlands. Selon les résultats inscrits dans les figures, nous avons remarqué que :

- L'incidence de BBTV dans la zone d'étude a été plus remarquée dans les hinterlands de la ville de Kindu. Cependant, l'incidence la plus élevée était notée aux sites : Pk16 Kalemba route Lokando avec 58,71\%, suivi de Pk10 Misenge avec 54,85\% et Pk7 Katako avec 53, $16 \%$.

- Par contre, cinq (5) cultivars ont été identifiés Kebobo, Mbudi, Muyahudi, Ndogila ET Nsekumuna. Ainsi, le cultivar Kebobo présente un taux plus élevé respectivement avec 48,6\% et le cultivar Mfuba Ndogila avec 47,87\%.

- La sévérité de BBTV dans la zone d'étude était abondant au niveau 2 (stries foncées jusqu'au pseudo-tronc) et niveau 4 (taille réduite de feuilles décolorées); néanmoins, les sites Alunguli Centre, Alunguli Sud Et Pk7 Katako route Kibombo ont présenté respectivement un taux de sévérité plus élevée respectivement avec $95,5 \%$ au niveau 3 et 86,66\% niveau 4 et $64,58 \%$ niveau 4.

- Ainsi, le cultivar ayant présenté un taux d'incidence la plus élevée par rapport à d'autres était le cultivar Nsekumuna avec $44 \%$ et une sévérité de $39,57 \%$ suivi des autres.

- A ce niveau les cultivateurs peuvent utiliser les cultivars MBUDI et MUYAHUDI Car ils ont été légèrement résistants au BBTD par rapport aux autres cultivars.

Suite aux résultats obtenue au cours de cette recherche, nous lançons un SOS aux autorités de la place afin d'organiser les campagnes de sensibilisation pour la lutte contre ce virus de BBTD (banana bunchy top disease) ainsi de promouvoir la culture de bananier dans le souci de maintenir la loi de l'offre et de la demande des produits au marché. Pour les chercheurs, nous leurs demandons de continuer avec cette étude suivant le temps et le milieu, tout en introduisant d'autres aléas que nous n'avions pas utilisés, tels que la température, le climat, le vent, sur le degré de propagation de la maladie ainsi que se mettre au travail sur les méthodes pour éviter la maladie comme par exemple « la macro propagation de rejets de bananier (PIF) » pour approvisionner les matériels aux agriculteurs qui utilisent les matériels tout venant chose qui influence la propagation de la maladie et qui occasionne la baisse de leur rendement.

\section{REFERENCES}

1. M.Jenny, C. Uhl, C. Roche, I. Duluc, V, Guillermin, F. Guillemot , J. Jensen, M, Kedinger, G.Gradwohl, Neurogenin3 is differentially required for endocrine cell fate specification in the intestinal and gastric epithelium, EMBO J. 2002 Dec 2;21(23):6338-47. doi: 10.1093 /emboj /cdf649.

2. P.Y.Teycheney, B.E.L.Lockhart, I.Acina and T. Candresse; Detection of Banana mild manioc virus and Banana virus $x$ by polyvalent degenerated olizo nucleotide PT-PCP (PDO-RP-PCP).Journal of biological Methods, 2007, 142:41-49. 
3. R.Swennen. Et D. Vuylsteke , Bananier. In: H.R.Raemarkers (éd.). Agriculture en Afrique tropicale. DGCI, Bruxelles, 2001, 611-636.

4. FAO, Travailler ensemble pour lutter contre les maladies de la banane en Afrique Subsaharienne, 2006.

5. Anonyme, Atelier sur la virose et la fusariose à Xanthomonas du bananier : Relever les défis des menaces des maladies émergentes à la banane et les stratégies de sensibilisation, de surveillance et de gestion de ces maladies en Afrique subsaharienne 24- 28 Août 2009, Arusha, Tanzanie,2009.

6. K. Bakelena et K. Mankangidila, La production bananière au Zaïre, Info-musa,1996, 5, 2. 20-22.

7. Mobambo Cité par Bangata, cours de Phytotechnie spécial, université de Kinshasa faculté des sciences agronomiques Ier grade phyto 2021 cours inédit.

8. Anonyme, Rapport mensuel, service de météorologie de l'aéroport de Kindu,2020

9. FAOSTAT, Food and agricultural commodities by country, 2009. FAO, Rome.Availabe on-line ['http: //www.faostat.fao.org/site/339/default.aspx'

10. CRBP, Etude économique et amélioration de la filière plantain. Rapport d'activités scientifiques annuelles, Njombé. Cameroun, 1986, 185-192.

11. C.Mukala, cours de phytopathologie et zoologie agricole spéciale et cours de relation plant et hôte, 2021. UPEN/Kinshasa/DES cours inédit ;

12. INIBAP: International Network for the Improvement of Banana and Plantain. 2000 (Banana: food for the poor. INIBAP, Montpellier, France.)

13. A.Josette, A. Souzanne, L. Herve , Principe de virologie végétale: pouvoir pathogène, écologie des virus. INRA. Editions, 2001, 408 p.

14. M.L.Caruana, Analyse du risque phytosanitaire (ARP). Bananiers. Banana Bunchy Top babuvivus. CIRAD.2003, P31.

15. F.Ngama , phytopathologie et zoologie agricole spéciale $\left(1^{\mathrm{er}}\right.$ grade), cours inédit, FSA/UNIKI (RDC)2016 ;

16. www.Maniema.climate-data.org: KOPPEN; https.climate-data.org/Afrique/CongoKinshasa/Maniema-1548/

17. https://www.sifee.org/home 2015: climat et secrétariat international francophone pour l'évaluation de l'environnement/ Kindu Maniema 12p

18. M'PIANA, cours de climatologie et le cours de géologie, géomorphologie et minéralogie cours de Ier graduat université de Kindu faculté des sciences agronomiques cours inédits ,2010

19. Anonyme, Rapport mensuel, service de météorologie de l'aéroport de Kindu,2020

20. YANNICK, Biogéographie, 2ème grade, cours inédit, FSA/UNIKI (RDC),2018

21. M.A.Kalonji, Principes de Phytopathologie, 2008, 36-111pp. 
22. G. Monde ; J. Walangululu,\& C. Bragard, Screening cassava for resistance to cassava mosaic disease by grafting and whitefly inoculation; Archives of Phytopathology and Plant Protection , 2012,45, 18 ,2189-2201https:// doi.org/10.1080 /03235408. 2012. 724969

23. M.Roland; Le végétal est le vaccin mental à cette crise sanitaire ,2021p6

24. Thomas J.E. Iskara, M.L. Carana, Bunchy top; in JONES D.R (ed.) :Disease of banana, Abaca and Ensete. London, CABI, Tropical Pest Management, 1999, 36 -3): 249257.

\section{* Corresponding Author: SHADARI SALUMU Al hadid}

Département de Phytotechnie, Faculté des Sciences Agronomiques, Université de Kindu (UNIKI/Maniema/RD Congo).Université de Kindu UNIKI, République Démocratique du Congo (RDC)/ ville de Kindu province du Maniema

Online date of publication: 25.10.2021 